\title{
ADAPTACIÓN, RESISTENCIA MÚLTIPLE A ENFERMEDADES Y TOLERANCIA A SUELOS ÁCIDOS EN GENOTIPOS DE FRIJOL ${ }^{1}$
}

\author{
Ernesto López ${ }^{2}$, Oscar Hugo Tosquy², Bernardo Villar ${ }^{3}$, Enrique Noe Becerra ${ }^{2}$, Francisco Javier Ugalde ${ }^{2}$
}

\begin{abstract}
RESUMEN
Adaptación, resistencia múltiple a enfermedades y tolerancia a suelos ácidos en genotipos de frijol. El objetivo del presente trabajo fue seleccionar genotipos de frijol con alto potencial de rendimiento, amplia adaptación, resistencia múltiple a enfermedades y tolerancia a suelos ácidos. Para lo cual, durante el ciclo otoño-invierno 1999-2000, se estableció un experimento uniforme de rendimiento de 16 genotipos incluyendo al testigo Negro Jamapa en cuatro ambientes en Veracruz y Chiapas, México. Se utilizó el diseño experimental de bloques completos al azar con tres repeticiones. Se evaluó el rendimiento de grano mediante análisis de varianza individual, combinado y parámetros de estabilidad. Los datos de reacción a enfermedades se cuantificaron con una escala de 19. Para evaluar la tolerancia a suelos ácidos se utilizó el Índice de Eficiencia (IE). Los resultados de rendimiento de grano y reacción a enfermedades, señalaron diferencias significativas para localidades, genotipos y la interacción localidad-genotipo; mientras que para suelos ácidos no hubo significancia entre localidades y la interacción. Los mejores genotipos en cuanto a rendimiento, adaptación y tolerancia a suelos ácidos fueron Negro 8025, DOR-454, Icta Ju 95-112, Icta Ju 93-15 y DOR-448, de los cuales el primero y el último presentaron resistencia múltiple a enfermedades. Las líneas UCR-55, TLP22 y DOR-500, mostraron también buenos rendimientos y resistencia múltiple a enfermedades.
\end{abstract}

\begin{abstract}
Adaptation, multiple disease resistance and acid soils tolerance of bean genotypes. The objective of this experiment was to select bean genotypes with high yield potential, wide adaptation, multiple disease resistance and tolerance to acid soils. For that reason, during the Fall-Winter 1999-2000 cycle, a uniform yield trial was conducted in four environments in Veracruz and Chiapas, Mexico. Sixteen genotypes were evaluated, including the varieties Negro Jamapa as a regional control. A randomized Complete Block design with three replications was used. Grain yield was evaluated by means of individual and combined analysis of variance and stability parameters. Reaction to diseases was graded according to a 1-9 scale; the Efficiency Index (IE) was used to evaluate the tolerance to acid soils. There were significant differences in grain yield and tolerance to diseases for localities, genotypes and the locality -genotype interaction; whereas for the tolerance to acid soils there was no significant differences between localities and the interaction. The best genotypes with regard to yield, adaptation and tolerance to acid soils were Negro 8025, DOR-454, Icta Ju 95-112, Icta Ju 93-15 and DOR-448; the first and the last varieties had multiple resistance to diseases. The lines UCR-55, TLP-22 and DOR-500 also showed high yields and multiple disease resistance.
\end{abstract}

\section{INTRODUCCIÓN}

En los estados de Veracruz y Chiapas, México, se siembran 40 mil y 110 mil hectáreas de frijol, respectivamente. Estas entidades son las de mayor superficie en el sureste de México (SAGAR 1997), y en ambas, los problemas bióticos como abióticos del cultivo de frijol, son muy similares. Entre los primeros destacan las plagas como: mosca blanca (Bemisia tabaci), diábroticas (Diabrotica balteata LeConte) y chicharritas (Empoasca kraemeri Ross and Moore), así como las enfermedades mosaico dorado amarillo (BGYMV), roya (Uromyces

\footnotetext{
1 Recibido para publicación el 30 de agosto del 2003. Trabajo presentado en la XLVIII Reunión del PCCMCA, celebrado en República Dominicana. 2002.

2 INIFAP- Campo Cotaxtla., Ap. 429, Veracruz Ver., México. salinaser@hotmail.com

3 INIFAP-Campo Centro de Chiapas., Ap. Núm. 1, Ocozocoautla ,Chiapas, México.
} 
appendiculatus var. appendiculatus), mancha angular (Phaeoisariopsis griseola) y antracnosis (Colletotrichum lindemuthianum), mientras que entre abióticos, la sequía y los suelos ácidos de baja fertilidad (López y Acosta 1998). El Programa de mejoramiento de frijol del Campo Cotaxtla del INIFAP, ha liberado variedades con amplia adaptación, alto rendimiento y con resistencia a enfermedades como Negro Huasteco-81, Negro Cotaxtla 91, Negro INIFAP y Negro Tacaná (Yoshii et al. 1987, López y Rodríguez 1992, Villar y López 1993, López et al. 1994 y López et al. 1997). Además de lo antes mencionado, algunas presentan tolerancia a factores abióticos como Negro Huasteco-81, a la sequía terminal (Janssen et al. 1989), y la variedad Negro INIFAP, tolerancia a suelos ácidos de baja fertilidad (López et al. 1998a). Con relación a las deficiencias de nutrimentos en el suelo, las cuales son muy comunes en la región, se tienen referencias de disponibilidad de germoplasma con adaptación a estas condiciones (Thung 1991, Lynch y Beebe 1994, Worthmann 1994, López et al. 1995, 1998b). En este sentido, es muy importante que los rendimientos del frijol se incrementen y estabilicen en el trópico húmedo. Debido a que el cultivo ha co-evolucionado con sus patógenos asociados (Guzmán et al. 1996), se requiere la acumulación de genes de resistencia a éstos; así como tener una mayor adaptación a los diversos factores abióticos que limitan su producción. Por lo anterior, el objetivo del presente trabajo fue identificar genotipos de frijol con amplia adaptación y rendimiento en el trópico húmedo de México y con resistencia a factores bióticos y abióticos.

\section{MATERIALES Y MÉTODOS}

Durante el ciclo agrícola otoño-invierno 19992000, se condujeron cuatro Ensayos Uniformes de Rendimiento (EUR) de frijol, bajo diferentes condiciones de humedad, suelo y aplicación de macronutrimentos. Tres se realizaron en Veracruz (uno en Medellín de Bravo, a $18^{\circ} 44^{\prime} \mathrm{LN}, 95^{\circ} 58^{\prime} \mathrm{LO}$ y $16 \mathrm{msnm}$, y dos en Isla a $18^{\circ} 06^{\prime} \mathrm{LN}, 95^{\circ} 32^{\prime} \mathrm{LO}$ y $65 \mathrm{msnm}$ ) y uno en el centro de Chiapas (Ocozocoautla $16^{\circ} 46^{\prime} \mathrm{LN}, 9^{\circ} 27^{\prime} \mathrm{LO}$ y $846 \mathrm{msnm})$. En los ensayos se incluyeron 16 genotipos de frijol; incluyendo a la variedad Negro Jamapa como el testigo local, por ser la variedad más sembrada en los dos estados. En todos los ensayos se utilizó el diseño de bloques completos al azar con tres repeticiones y las parcelas experimentales fueron de tres surcos de $5,0 \mathrm{~m}$ de longitud, con separación de $60 \mathrm{~cm}$. El rendimiento se calculó en $\mathrm{kg} / \mathrm{ha}$ y se ajustó a $14 \%$ de humedad. Los datos se sometieron a un análisis combinado de los cuatro ensayos y se compararon las medias de tratamientos por la prueba DMS a un nivel de signifi- cancia del 0,05. También se realizó un análisis de regresión con los cuatro ensayos por el método de Eberhart y Russell (1966); y la adaptación y estabilidad de las líneas se clasificaron con base al coeficiente y a la desviación de la regresión (Carballo y Márquez 1970).

Las evaluaciones de la reacción a las enfermedades (antracnosis, roya y mancha angular), se realizó en las localidades de Isla y Medellín de Bravo, Veracruz, en ambas localidades se sembró a fines de octubre, debido a las fuertes lluvias ocurridas durante todo el ciclo del cultivo. Las enfermedades se evaluaron mediante la escala de 1-9 (CIAT 1987), cuyos valores son: 1-3= resistentes, 4-6 = intermedios y 7-9= susceptibles. Se efectuó el análisis de varianza de la incidencia de cada enfermedad y separación de medias por DMS al 0,05 de probabilidad; además se realizó un análisis combinado de las tres enfermedades que se presentaron (roya, antracnosis y mancha angular).

La respuesta de los genotipos bajo condiciones de suelo ácido, se evaluó en Isla, Veracruz, en suelo tipo Acrisol de pH 4,4, según clasificación FAO-UNESCO (Duadal 1968). Un ensayo fue con aplicación de 1,2 t ha $^{-1}$ de cal dolomítica al suelo, cantidad suficiente para elevar el pH a 5,5, y otro sin cal. La cal se incorporó al suelo, 30 días antes de la siembra. Los dos ensayos se fertilizaron con 20-20-0 de $\mathrm{N}$ y $\mathrm{P}$, respectivamente, al momento de la siembra. Para la evaluación de la respuesta en rendimiento de cada genotipo, se utilizó el índice de eficiencia (IE) propuesto por Graham (1984), a través de la ecuación siguiente: $\mathrm{IE}=(\mathrm{Ri}$ sin cal/promedio sin cal) ( $\mathrm{Ri}$ con cal/promedio con cal); donde $\mathrm{Ri}=$ rendimiento individual de cada genotipo. Este índice señala la respuesta promedio de cada genotipo bajo los dos tratamientos evaluados.

\section{RESULTADOS Y DISCUSIÓN}

Los mayores rendimientos por localidad fueron en Medellín de Bravo, Veracruz, bajo condiciones de riego; los rendimientos más bajos se obtuvieron en Isla, Veracruz, en suelo ácido y sin aplicación de cal dolomítica (Cuadro 1). En Isla se presentó la enfermedad de la antracnosis, la cual disminuyó considerablemente los rendimientos. En los análisis de los ensayos individuales se encontraron diferencias altamente significativas entre genotipos y en el análisis de conjunto las hubo entre genotipos, localidades y en la interacción de ambos factores. Los genotipos que mostraron los mayores rendimientos en promedio de las cuatro localidades fueron: Negro 8025, DOR-454, Icta Ju 95-12, Icta Ju 9315 y DOR-448, los cuales fueron estadísticamente 
iguales entre sí y superiores a la variedad testigo Negro Jamapa (Cuadro 2). La acción conjunta genotipo x localidad tuvo como mejores combinaciones a TLP-22, Icta Ju 95-112 y DOR-448, establecidos en la localidad de Cotaxtla bajo condiciones de riego con rendimientos superiores a las $2 \mathrm{t} / \mathrm{ha}$, aun cuando se tuvo roya y mancha angular en las etapas finales del cultivo, por lo cual no se afectó el rendimiento de grano.

Cuadro 1. Promedio de rendimiento de 16 genotipos de frijol en cuatro ambientes de Veracruz y Chiapas, México. Ciclo 1999-2000.

\begin{tabular}{lccc}
\hline \multicolumn{1}{c}{ Localidad } & Condición & $\begin{array}{c}\text { Rendimiento } \\
\text { kg/ha }\end{array}$ \\
\hline $\begin{array}{l}\text { Medellín de Bravo, } \\
\text { Veracruz }\end{array}$ & $\begin{array}{c}\text { Riego-NP-40-40-00 } \\
\text { Ocozocoautla, Chis. } \\
\text { Humedad Residual- NP- } \\
\quad \text { 40-40-00 }\end{array}$ & 751 & b \\
Isla, Veracruz & Humedad Residual + Cal- & 606 & b \\
& NP-20-20-00 & & \\
Isla, Veracruz & Humedad Residual sin Cal- & 586 & b \\
& NP-20-20-00 & & \\
Promedio & & 910 & \\
DMS 0,05 & & 307 & \\
\hline
\end{tabular}

Tratamientos con letras similares son estadísticamente iguales de acuerdo a DMS al 5\%.

En el mismo Cuadro 2, se presentan los valores de los parámetros bi y $\mathrm{S}^{2}$ di y la clasificación de cada uno de los genotipos con base al esquema propuesto por Carballo y Márquez (1970). Se puede apreciar que de los 16 genotipos evaluados para rendimiento en los cuatro ambientes, 11 resultaron estables en su respuesta (bi $\left.=1 ; \mathrm{S}^{2} \mathrm{di}=0\right)$; dentro de esta categoría se encontraron las líneas DOR-454, Icta Ju 95-112. Icta Ju 9315 , DOR-448, Dor-500 y la variedad Negro 8025 , junto con otros cinco genotipos. A su vez, tres materiales respondieron mejor en buenos ambientes consistentes (bi $>1$; $\mathrm{S}^{2} \mathrm{di}=0$ ): II-307, TLP-22 y UCR-55 y dos respondieron mejor en ambientes desfavorables consistentes (bi $<1 ; \mathrm{S}^{2} \mathrm{di}=0$ ): Icta Ligero y TLP-16, similares resultados a los reportados por López et al. (1999).

Con respecto a la variable reacción a enfermedades, en la localidad de Isla, además de la precipitación se presentaron temperaturas frescas y alta humedad relativa, las cuales fueron condiciones ideales para la ocurrencia de antracnosis, donde previamente se había identificado por medio de variedades diferenciales a las razas 9 y 73 de Colletotrichum lindemuthianum (López et al. 2000). En el caso de las otras dos enfermedades no se identificaron sus razas patogénicas.
Cuadro 2. Rendimiento promedio y parámetros de estabilidad de 16 genotipos de frijol en cuatro localidades de Veracruz y Chiapas, México. Ciclo 1999-2000.

\begin{tabular}{lrrrc}
\hline Genotipo & kg/ha & bi & \multicolumn{1}{c}{ S $^{2}$ di } & Clasificación \\
\hline Negro 8025 & 1.128 & 0,67 & $-18.448,11$ & $\mathrm{~A}$ \\
DOR-454 & 1.075 & 0,84 & $34.205,58$ & $\mathrm{~A}$ \\
Icta Ju 95-112 & 1.062 & 1,23 & $-30.950,08$ & $\mathrm{~A}$ \\
Icta Ju 93-15 & 1.060 & 1,12 & $-21.780,08$ & $\mathrm{~A}$ \\
DOR-448 & 1.031 & 1,18 & $-19.774,02$ & $\mathrm{~A}$ \\
II-307 & 1.006 & 1,30 & $-36.503,45$ & $\mathrm{E}$ \\
TLP-22 & 994 & 1,41 & $-34.559,02$ & $\mathrm{E}$ \\
UCR-55 & 974 & 1,24 & $-35.206,20$ & $\mathrm{E}$ \\
TLP-20 & 954 & 1,21 & $2.548,11$ & $\mathrm{~A}$ \\
TLP-18 & 914 & 0,82 & $-25.551,67$ & $\mathrm{~A}$ \\
NEPA-68 & 874 & 1,31 & $-29.647,70$ & $\mathrm{~A}$ \\
DOR-500 & 867 & 1,13 & $-33.638,58$ & $\mathrm{~A}$ \\
TLP-16 & 751 & 0,19 & $-28.555,16$ & $\mathrm{C}$ \\
NEPA-69 & 713 & 0,94 & $2.452,64$ & $\mathrm{~A}$ \\
Negro & & & & \\
Jamapa (T.L.) & 599 & 0,78 & $5.806,97$ & $\mathrm{~A}$ \\
Icta Ligero & 557 & 0,63 & $33.186,22$ & $\mathrm{C}$ \\
Promedio & 910 & & & \\
DMS 0,05 & 307 & & & \\
C.V. & 21,05 & & & \\
Variedades (V) & $* *$ & & & \\
Localidades (L) & $* *$ & & & \\
Interacción L x V & $* *$ & & & \\
\hline A = Estable & & & & \\
C = Responde mejor en ambientes & \\
E = Responde mejor en buenos ambientes consistente & \\
& & & & \\
\hline
\end{tabular}

Los análisis de varianza individuales detectaron diferencia altamente significativa para genotipos en la reacción a roya, mancha angular y antracnosis. Los genotipos resistentes a la roya fueron Negro 8025 y UCR55 (calificación < 3) y 11 resultaron intermedios en su reacción, mismo comportamiento de los trabajos realizados por López et al. $(1999,2001)$. Los genotipos tolerantes a la antracnosis fueron UCR-55; esta fue reportada como resistente por Araya (2000), en Costa Rica, así como TLP-22 y DOR-500 (calificación <4), mientras que la línea II-307 fue resistente a la mancha angular, similares a los resultados reportados por López et al. (2000). La roya fue la enfermedad de mayor severidad, mientras que las de menor fueron la antracnosis y la mancha angular.

El análisis de conjunto de las enfermedades (Cuadro 3), señaló diferencia significativa para resistencia múltiple a las enfermedades. La variedad UCR-55, resultó resistente, mientras que: TPL-22, Negro 8025. DOR-500 y DOR-448 (calificación < 4) fueron tolerantes. Estas 
Cuadro 3. Diferencias en la resistencia a enfermedades de 16 genotipos de frijol. Veracruz, México. Ciclo 19992000 .

\begin{tabular}{lcccccc}
\hline \multicolumn{1}{c}{ Genotipo } & ANT $^{\mathbf{1}}$ & ANT $^{\mathbf{2}}$ & Roya $^{\mathbf{3}}$ & $\mathbf{M A}^{\mathbf{4}}$ & $\mathbf{C C}^{\mathbf{5}}$ \\
\hline Negro Jamapa & 8,00 & 4,67 & 9,00 & 3,67 & 6,33 & $\mathrm{a}$ \\
TLP-16 & 5,00 & 3,33 & 6,00 & 6,33 & 5,17 & $\mathrm{ab}$ \\
TLP-20 & 4,00 & 3,33 & 4,67 & 5,00 & 5,08 & $\mathrm{ab}$ \\
Icta Ju 93-15 & 4,67 & 4,00 & 6,33 & 5,33 & 5,08 & $\mathrm{ab}$ \\
II-307 & 8,00 & 5,67 & 4,33 & 2,00 & 5,00 & $\mathrm{abc}$ \\
NEPA-68 & 4,67 & 4,00 & 6,00 & 5,00 & 4,83 & $\mathrm{abc}$ \\
Icta Ju 95-112 & 3,33 & 3,33 & 7,33 & 4,67 & 4,67 & $\mathrm{abc}$ \\
TLP-18 & 5,00 & 3,33 & 4,67 & 5,00 & 4,67 & $\mathrm{abc}$ \\
NEPA-69 & 3,67 & 4,33 & 5,33 & 6,33 & 4,58 & $\mathrm{abc}$ \\
DOR-454 & 4,33 & 4,00 & 4,00 & 4,33 & 4,17 & $\mathrm{abc}$ \\
DOR-448 & 4,67 & 3,33 & 3,67 & 4,33 & 4,00 & $\mathrm{bc}$ \\
DOR-500 & 3,33 & 3,00 & 4,00 & 4,67 & 3,83 & $\mathrm{bc}$ \\
Icta Ligero & 7,00 & 4,33 & 3,67 & 0,00 & 3,75 & $\mathrm{bc}$ \\
Negro 8025 & 6,00 & 2,67 & 1,67 & 3,67 & 3,50 & $\mathrm{bc}$ \\
TLP-22 & 2,67 & 3,33 & 4,33 & 3,00 & 3,33 & $\mathrm{bc}$ \\
UCR-55 & 2,33 & 3,33 & 2,33 & 3,00 & 2,83 & $\mathrm{c}$ \\
Promedio & 4,79 & 3,71 & 4,92 & 4,29 & 4,42 & \\
DMS 0,05 & 2,42 & 2,21 & 1,90 & 2,80 & 2,22 & \\
ANDEVA & $* *$ & $\mathrm{~ns}$ & $* *$ & $* *$ & $* *$ & \\
\hline
\end{tabular}

$1=$ Antracnosis, Isla $+\mathrm{Cal},{ }^{2}=$ Antracnosis, Isla $-\mathrm{Cal},{ }^{3}=$ Roya Campo Cotaxtla, ${ }^{4}=$ Mancha Angular Campo Cotaxtla, $5=$ Calificación conjunta.

mismas líneas y la variedad habían sido reportadas como resistentes y tolerantes por López et al. (1999), Acosta (2000). La variedad Icta Ligero presentó tolerancia en el análisis de conjunto a enfermedades, probablemente debido al escape a ellas por su precocidad como es el caso de la mancha angular y roya, las cuales se presentaron en la fase reproductiva del cultivo.

La respuesta en rendimiento de las variedades en condiciones de suelo ácido, así como el Índice de Eficiencia (IE), se presentan en el Cuadro 4. El análisis de varianza mostró diferencia significativa entre genotipos, pero entre localidades (con cal y sin cal) e interacción, no hubo diferencia significativa, probablemente debido a la presión por antracnosis que pudo enmascarar las diferencias entre aplicar cal y no aplicar. Los genotipos que obtuvieron los mayores rendimientos de tratamientos sin y con cal fueron: Negro 8025, DOR-454, ICTA Ju 93-15 y DOR-448, con índices de eficiencia agronómica 2,$75 ; 2,53 ; 1,68$ y 1,60 , respectivamente, los cuales superaron en ambos parámetros al testigo Negro Jamapa cuyo índice fue de 0,16 . Los datos demuestran que el programa de frijol de México dispone o cuenta con germoplasma con diferentes niveles de tolerancia a suelos ácidos que puedan representar una alternativa tecno-
Cuadro 4. Rendimiento promedio de 16 genotipos de frijol sembrados con y sin cal en un suelo con pH 4,4 de Isla, Veracruz, México. Veracruz, México. Ciclo 1999-2000.

\begin{tabular}{lrrr}
\hline \multicolumn{1}{c}{ Genotipos } & $\begin{array}{c}\text { Con cal } \\
\mathbf{k g} / \mathbf{h a}\end{array}$ & $\begin{array}{c}\text { Sin cal } \\
\mathbf{k g} / \mathbf{h a}\end{array}$ & IEi \\
\hline Negro 8025 & 1.080 & 963 & 2,75 \\
DOR-454 & 964 & 930 & 2,53 \\
ICTA Ju 93-15 & 786 & 755 & 1,68 \\
DOR-448 & 763 & 746 & 1,60 \\
Icta Ju 95-112 & 653 & 686 & 1,26 \\
UCR-55 & 640 & 663 & 1,20 \\
TLP-16 & 627 & 591 & 1,05 \\
TLP-18 & 619 & 585 & 1,02 \\
II-307 & 593 & 561 & 0,94 \\
TLP-22 & 589 & 549 & 0,90 \\
DOR-500 & 547 & 531 & 0,82 \\
NEPA 68 & 463 & 498 & 0,62 \\
TLP-20 & 448 & 383 & 0,48 \\
Icta Ligero & 394 & 380 & 0,42 \\
NEPA 69 & 317 & 313 & 0,27 \\
Negro Jamapa (T.L.) & 265 & 230 & 0,16 \\
\hline
\end{tabular}

lógica para las áreas frijoleras de Isla, Veracruz (López et al. 1995, 1998a). Por otra parte, todos los genotipos con IE menores a la unidad son los que presentaron pobre adaptación y susceptibilidad a la antracnosis; ya que su rendimiento fue menor al promedio.

\section{CONCLUSIONES}

Negro 8025, DOR-454, Icta Ju 95-112, Icta Ju-9315 y DOR-448, presentaron alto rendimiento, estabilidad y tolerancia a suelos ácidos. Negro 8025 y DOR448, también presentaron resistencia múltiple a enfermedades.

UCR-55 y TLP-22 y DOR-500 mostraron resistencia múltiple a las enfermedades. Estos materiales se pueden utilizar como progenitores en los programas de mejoramiento de frijol.

\section{LITERATURA CITADA}

ACOSTA, A. J.; ROSALES, R.; PÉREZ, P. 2000. Negro 8025 nueva variedad de frijol negro para valles altos de México. Desplegable. No. 1. INIFAP. Campo Experimental Valle de México. Chapingo, Edo. De México.

ARAYA, R. 2000. Nueva variedad de frijol negro para Costa Rica. In: XLVI Reunión Anual del PCCMCA (Resúmenes). San Juan, Puerto Rico. p. 65. 
CENTRO INTERNACIONAL DE AGRICULTURA TROPICAL (CIAT). 1987. Sistema estándar para evaluación de germoplasma de frijol. Schoonhoven; A. Van y Pastor-Corrales, M.A. (comps.). Calí Colombia. 56 p.

CARBALLO, A.; MARQUÉS, F. 1970. Comparación de variedades de maíz del Bajío y de la mesa central por su rendimiento y estabilidad. Agrociencia 5 (1): 129-146

EBERHART, A.; RUSSELL, A. 1966. Stability parameters for comparing varieties. Crop, Science 6: 36-40.

DUDAL , R. 1968. Definition of soil its for the soil map of the world. FAO. Rome. World resource report 33. 96 p.

GRAHAM, R. D. 1984. Breeding for nutritional characteristics in cereals. Advances in Plant Nutrition 1:57-102.

GUZMAN, P.; GILBERTSON, L.; NODARI, R.; JOHNSON, C.; TEMPLE, S.; MANDALA, D.; MAHANDAWIRE, C.; GEPTS, P. 1996. Characterization of variability in the fungus Phaoisariopsis griseola suggests co-evolution with the common bean (Phaseolus vulgaris) Phytopathology 85: 600-607.

JANSSEN, W.; LUNA, A.C.; LÓPEZ E. 1999. Adopción de la variedad Negro Huasteco-81, en las Huastecas de México. Documento Interno S/P. CIAT-INIFAP. Calí, Colombia. 32p.

LÓPEZ, E.; ACOSTA, A. J.; BECERRA, N. E.; FRAIRE, G.; OROZCO, S.; BEEBE, S. 1997. Registration of Negro Tacaná Common Bean. Crop Science: 37(3): 1022.

LÓPEZ E.; ACOSTA, A. J. 1998. Mejoramiento de frijol negro mesoamericano en el trópico de México. In: R. Lépiz (ed.). Memoria Taller Internacional de Mejoramiento Genético de Frijol Negro Mesamericano. Veracruz, Veracruz, México. p. 15-16.

LÓPEZ, E.; CUMPIAN, J.; UGALDE, F. J.; CANO, O. 1998a. Parcelas de Validación de frijol en suelos ácidos de Isla, Veracruz. In: G. Díaz (ed.). XI Reunión Científica-Tecnológica Forestal y Agropecuaria. Veracruz, Veracruz, México. p. 145-148.

LÓPEZ, E.; CUMPIAN, J.; CANO, O. 1998b. Tolerancia del frijol a suelos ácidos en Isla, Veracruz. In: P. Ramírez, F. Zavala, A. Mejía (eds.). Memoria del XVIII Congreso de Fitogenética: notas científicas. SOMEFI. Chapingo, México. p. 447.

LÓPEZ, E.; CUMPIAN, J.; DURÁN, A.; ACOSTA, A. J. 1995. Tolerancia del frijol común a baja disponibilidad de fós- foro en suelos de Veracruz, México. In: Taller Internacional sobre bajo fósforo en fríjol común. Araya, R. y Beck, D. (eds). San José, Costa Rica. p. 34-40.

LÓPEZ, E.; RODRÍGUEZ, R. 1992. Negro Cotaxtla-91: Nueva variedad de frijol para zonas tropicales de Veracruz. CECOT-CIRGOC-INIFAP. Folleto Técnico Num. 1. 8p.

LÓPEZ, E.; VILLAR, B.; CANO, O. 1994. Rendimiento y adaptación del frijol negro E-44 en el Trópico Húmedo de México. Revista Fitotecnia Mexicana 17(1): 39-47.

LÓPEZ, E.; ACOSTA, J. A.; CANO, O.; FRAIRE, G.; CUMPIAN, J.; BECERRA, N. E.; VILLAR, B.; UGALDE, F.J. 1999. Estabilidad de rendimiento de la línea de frijol negro DOR-500 en el Trópico Húmedo de México. Agron. Mesoam. 10(2): 69-74.

LÓPEZ, E.; ACOSTA, A. J.; AWALE, E.; KELLY, J. 2000. An unusual outbreak of anthracnose in the lowlands of Veracruz, México. An. Rep. Bean Improv. Coop. 43: 182-183.

LÓPEZ, E.; CANO, O.; VILLAR, B.; CUMPIAN, J.; UGALDE, J. F.; LÓPEZ, O. V. 2001. Evaluación de líneas de frijol negro en Veracruz y Chiapas, México. Agron. Mesoam. 12(2): 129-133.

LYNCH, J.; BEEBE, S. 1994. Adaptation of beans (Phaseolus vulgaris L.) to low phosphorus availability. HorScience 30(6): 1165-1171.

SAGAR. 1997. Frijol: Estimación de cosecha de primaveraverano 1997. (Documento de discusión interna). Subsecretaria de Agricultura y Ganadería. Dirección General de Agricultura. México, D.F. 15p.

THUNG, M. 1991. Bean agronomy in monoculture. In: Common bean research for crop improvement. Schoonhoven, A. Van and Voysest, O. (eds.). CAB International and CIAT. p. 737-834.

VILLAR, B.; LÓPEZ, E. 1993. Negro INIFAP: Nueva variedad de frijol para Chiapas y regiones similares. Revista Fitotecnia Mexicana 16 (2): 208-209.

WORTHMANN, C. S. 1994. Bean improvement for low fertility meeting, Kampala, Uganda. Network on Bean Research in Africa. CIAT. Cali, Colombia. Workshop series No. 25. pp. 2-5.

YOSHII, K.; RODRÍGUEZ, J.; NÚÑEZ, S.; CARRIZALES, N.; IBARRA, J. F.; PEREZ, P. 1987. Rendimiento y adaptación de la línea D-145 tolerante al mosaico dorado del frijol en el Sureste de México. Revista Fitotecnia Mexicana 9: 57-63. 